\title{
Possible Evidence for a Chiral Axial-Vector State in the $D$ Meson System
}

\author{
Kenji Yamada*, Muneyuki Ishida 9 , Shin Ishida ${ }^{\dagger}$, Daiki Ito $^{\dagger}$, \\ Toshihiko KomadA* and Hiroshi TonookA ${ }^{\dagger}$ \\ *Department of Engineering Science, Junior College Funabashi Campus \\ Nihon University, Funabashi 274-8501, Japan \\ I Department of Physics, Tokyo Institute of Technology, Tokyo 152-8551, Japan \\ ${ }^{\dagger}$ Atomic Energy Research Institute, College of Science and Technology \\ Nihon University, Tokyo 101-8308, Japan
}

\begin{abstract}
We reanalyze the $D^{*+} \pi^{-}$mass spectrum from CLEO II by the VMW method in order to examine the existence of a chiral axial-vector state, which is predicted in a covariant levelclassification scheme recently proposed, other than normal orbitally-excited $P$-wave states in the $D$ meson system. A result of the present analysis seems to suggest that there exists an extra axial-vector meson, in addition to the two normal ones, in a similar mass region.
\end{abstract}

\section{$\S 1 . \quad$ Introduction}

In the constituent quark model, together with heavy quark symmetry, the lowestlying positive parity excitations of heavy-light $Q \bar{q}$ meson systems are expected, in the limit $m_{Q} \rightarrow \infty$, to be two degenerate spin doublets with the total angular momentum $j_{q}=1 / 2$ and $3 / 2$ of the light quark, that is, four orbitally-excited states with $L=1$ labeled as

$$
\begin{aligned}
{ }^{j_{q}} L_{J} & ={ }^{1 / 2} P_{0},{ }^{1 / 2} P_{1} & \text { for the } j_{q}=\frac{1}{2} & \text { doublet, } \\
& ={ }^{3 / 2} P_{1},{ }^{3 / 2} P_{2} & \text { for the } j_{q}=\frac{3}{2} & \text { doublet. }
\end{aligned}
$$

In this limit heavy quark symmetry further requires that the $j_{q}=1 / 2$ states decay to ${ }^{1 / 2} S_{0}+\pi$ or ${ }^{1 / 2} S_{1}+\pi$ only in an $S$-wave, while the $j_{q}=3 / 2$ states decay only in a $D$-wave. It is therefore expected that the decay widths of the $j_{q}=1 / 2$ and $3 / 2$ states are broad and narrow, respectively.

On the one hand, a covariant level-classification scheme of quark-antiquark meson systems has been proposed, which gives them a covariant quark representation with definite Lorentz and chiral transformation properties. (1) In this scheme, assuming that chiral symmetry for the light-quark component in heavy-light meson systems is effective, the existence of extra scalar and axial-vector states is predicted, respectively, as chiral partners of the ground-state pseudoscalar and vector mesons. These what we call chiral scalar and axial-vector mesons are distinguished from the above-mentioned $P$-wave states, since the chiral scalar state is an analogue of the $\sigma(400-600)$ meson, which is difficult to be interpreted as the ${ }^{3} P_{0}$ state, as a chiral partner of the $\pi$ meson in the light-quark system.

In this report we present a possible evidence for the chiral axial-vector state, $D_{1}^{\chi}$, in the $D$ meson system. 


\section{$\S 2$. Reanalysis of the $D^{*+} \pi^{-}$mass spectrum from CLEO II}

We reanalyze the $D^{*+} \pi^{-}$mass spectrum, published by CLEO Collaboration, 2) by the VMW method in which the production amplitude is expressed by a sum of Breit-Wigner amplitudes for relevant resonances.

In the present analysis we take into account the four states $D_{2}^{*}\left({ }^{3 / 2} P_{2}\right), D_{1}\left({ }^{3 / 2} P_{1}\right)$, $D_{1}^{*}\left({ }^{1 / 2} P_{1}\right)$ and $D_{1}^{\chi}$ which can decay to $D^{*} \pi$. Then, following the VMW method, the production amplitude is given by

$$
\begin{gathered}
|A(s)|^{2}=\left|r_{1} e^{i \theta_{1}} \Delta_{D_{1}^{\chi}}(s)+r_{2} e^{i \theta_{2}} \Delta_{D_{1}^{*}}(s)\right|^{2}+\left|r_{3} e^{i \theta_{3}} \Delta_{D_{1}}(s)\right|^{2}+\left|r_{4} e^{i \theta_{4}} \Delta_{D_{2}^{*}}(s)\right|^{2}, \\
\Delta_{R}(s)=\frac{-m_{R} \Gamma_{R}}{s-m_{R}^{2}+i m_{R} \Gamma_{R}},
\end{gathered}
$$

where $r_{1}, \ldots, r_{4}$ and $\theta_{1}, \ldots, \theta_{4}$ are the production couplings and phases of respective resonances, and we assumed that $D_{1}^{\chi}$ and $D_{1}^{*}$ decay only through an $S$-wave, while $D_{1}$ only through a $D$-wave. The background $D^{*} \pi$ mass distribution is fit with a five-parameter threshould function given by

$$
\begin{gathered}
B G=\alpha(\Delta M)^{\beta} \exp \left[-\gamma_{1} \Delta M-\gamma_{2}(\Delta M)^{2}-\gamma_{3}(\Delta M)^{3}\right], \\
\Delta M=M\left(D^{*} \pi\right)-m_{D^{*}}-m_{\pi},
\end{gathered}
$$

where the parameters $\alpha, \beta, \gamma_{1}, \gamma_{2}$ and $\gamma_{3}$ are fixed through the fit to the total $D^{*} \pi$ mass spectrum.

Using the above production amplitude and background, we fit the $D^{*} \pi$ mass spectrum in the following three cases:

(a) High-mass $D_{1}^{*}$ with a mass of $2500<m_{D_{1}^{*}}<2600$ in $\mathrm{MeV}$,

(b) Low-mass $D_{1}^{*}$ with a mass of $2350<m_{D_{1}^{*}}<2500$ in $\mathrm{MeV}$,

(c) No $D_{1}^{\chi}$ and $D_{1}^{*}$.

Here the case (c) corresponds to the original analysis by CLEO Collaboration, though the background parametrization is somewhat different. The results of fits are shown in Fig. 1 and obtained values of the resonance parameters are given in Table I. In both the fits with high- and low-mass $D_{1}^{*}$, it is found that the mass and width of $D_{1}^{\chi}$ are $\approx 2310 \mathrm{MeV}$ and $\approx 20 \mathrm{MeV}$, respectively, and those of $D_{1}$ and $D_{2}^{*}$ are similar to the values reported so far. For the mass and width of $D_{1}^{*}$ we obtain $\approx 2600 \mathrm{MeV}$ and $\approx 200 \mathrm{MeV}$ in the high-mass fit, while $\approx 2420 \mathrm{MeV}$ and $\approx 200 \mathrm{MeV}$ in the low-mass fit.

In all the three cases of fits the $\chi^{2} / N_{\text {dof }}$ is best for the high-mass $D_{1}^{*}$ fit, though they are not so different from each other. It would be worth while noting that the two cases of fits with $D_{1}^{\chi}$ seem to describe the data better than the fit without $D_{1}^{\chi}$ in the mass region $2.15-2.5 \mathrm{GeV}$, where there appears to be an excess of data events at the mass $2.31-2.33 \mathrm{GeV}$.

\section{$\S 3 . \quad$ Theoretical remarks on the results}

We consider the mass splitting and mixing of $P$-wave meson multiplets, based on the Breit-Fermi Hamiltonian with vector-gluon and long-range-scalar exchange, 

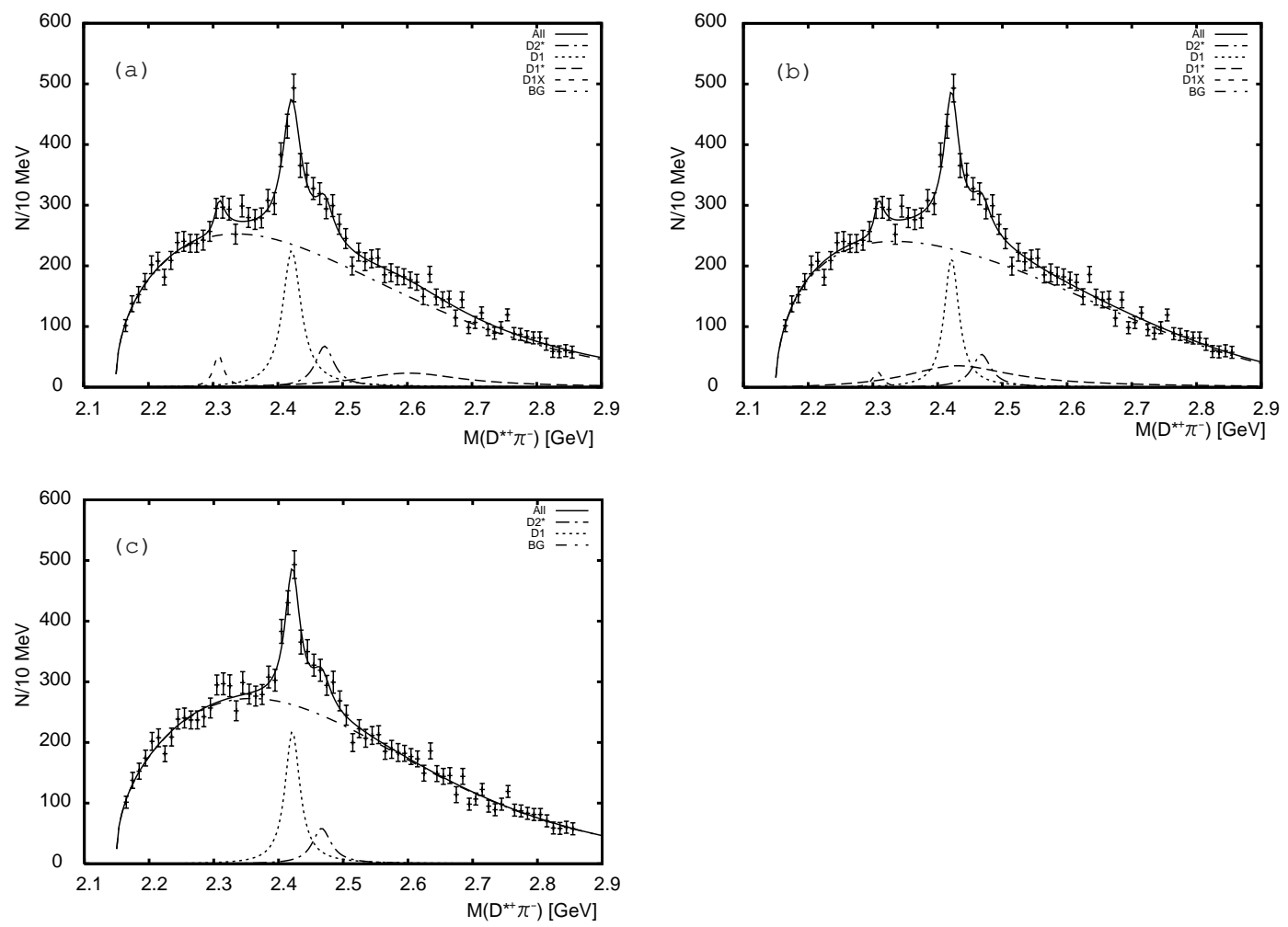

Fig. 1. The results of the fits to the $D^{*+} \pi^{-}$mass spectrum with (a) high-mass $D_{1}^{*}$, (b) low-mass $D_{1}^{*}$, and (c) no $D_{1}^{\chi}$ and $D_{1}^{*}$.

Table I. Values of the resonance parameters and $\chi^{2} / N_{\text {dof }}$ obtained from the respective fits.

\begin{tabular}{ccccccc}
\hline \hline & \multicolumn{2}{c}{ (a) Fit with high-mass $D_{1}^{*}$} & (b) Fit with low-mass $D_{1}^{*}$ & \multicolumn{2}{c}{ (c) Fit without $D_{1}^{\chi}$ and $D_{1}^{*}$} \\
\cline { 2 - 7 } State & Mass & Width & Mass & Width & Mass & Width \\
$(\mathrm{MeV})$ & $(\mathrm{MeV})$ & $(\mathrm{MeV})$ & $(\mathrm{MeV})$ & - & - \\
\hline$D_{1}^{\chi}$ & 2308 & 18.7 & 2307 & 17.4 & - & - \\
$D_{1}^{*}$ & 2596 & 199 & 2421 & 199 & - & 27.5 \\
$D_{1}$ & 2421 & 34.5 & 2421 & 27.0 & 2421 & 35.0 \\
$D_{2}^{*}$ & 2472 & 35.0 & 2468 & 35.0 & 2466 & $66.2 / 59$ \\
\hline$\chi^{2} / N_{\text {dof }}$ & $57.7 / 52$ & $58.0 / 52$ & & - \\
\hline
\end{tabular}

where we ignore the $P$-wave $D_{1}$ and $D_{1}^{*}$ states mixing with the chiral $D_{1}^{\chi}$ state. Taking a static potential due to single vector-gluon exchange to be $-4 \alpha_{s} / 3 r$, the spin-dependent part of the Hamiltonian for $P$-wave states can be expressed, to first order $1 / m_{Q}$, as

$$
\delta \mathcal{H}=C_{q} \mathbf{L} \cdot \mathbf{S}_{q}+C_{Q}\left(\mathbf{L} \cdot \mathbf{S}_{Q}+S_{T}\right), S_{T}=3\left(\mathbf{S}_{q} \cdot \hat{\mathbf{r}}\right)\left(\mathbf{S}_{Q} \cdot \hat{\mathbf{r}}\right)-\mathbf{S}_{q} \cdot \mathbf{S}_{Q}
$$


with

$$
\begin{aligned}
C_{q} & =\left(\frac{1}{2 m_{q}^{2}}+\frac{1}{m_{q} m_{Q}}\right)\left\langle\frac{4 \alpha_{s}}{3 r^{3}}\right\rangle-\frac{1}{2 m_{q}^{2}}\left\langle\frac{1}{r} \frac{d V_{s}}{d r}\right\rangle, \\
C_{Q} & =\frac{1}{m_{q} m_{Q}}\left\langle\frac{4 \alpha_{s}}{3 r^{3}}\right\rangle,
\end{aligned}
$$

where $V_{s}(r)$ is the static potential due to long-range-scalar exchange and the spinspin interaction is neglected because of its contact nature. The Hamiltonian $\delta \mathcal{H}$ gives rise to the mass splittings among $P$-wave multiplets and the mixing between the ${ }^{3 / 2} P_{1}$ and ${ }^{1 / 2} P_{1}$ states. Treating $\delta \mathcal{H}$ as a first-order perturbation and using the mass values of $D_{2}^{*}, D_{1}$ and $D_{1}^{*}$ obtained in the high-mass $D_{1}^{*}$ fit, we find $\approx 2470$ $\mathrm{MeV}$ for the mass of $D_{0}^{*}\left({ }^{1 / 2} P_{0}\right)$ and $\phi-\phi_{H Q} \approx-3.64^{\circ}$ for the deviation of the $D_{1}\left({ }^{3 / 2} P_{1}\right)-D_{1}^{*}\left({ }^{1 / 2} P_{1}\right)$ mixing angle from the heavy-quark-symmetry limit with the parameter values of the unperturbed mass $M_{0}=2490 \mathrm{MeV}$ common to all four states, $C_{q}=-73.81 \mathrm{MeV}$ and $C_{Q}=47.15 \mathrm{MeV}$, where we have chosen a solution with $C_{Q}>0$ in accord with the above definition of $C_{Q}$. For the low-mass $D_{1}^{*}$ case of fits there is no solution.

\section{$\S 4$. Concluding remarks}

We have reanalyzed the $D^{*+} \pi^{-}$mass spectrum published by CLEO Collaboration and found a possible evidence for the chiral axial-vector meson $D_{1}^{\chi}$ with a mass and width of $\approx 2310 \mathrm{MeV}$ and $\approx 20 \mathrm{MeV}$, respectively. We have also found the mass and width of $D_{1}^{*}$ to be $\approx 2600 \mathrm{MeV}$ and $\approx 200 \mathrm{MeV}$, together with similar masses and widths of $D_{1}$ and $D_{2}^{*}$ to those reported so far, among which the spin-dependent Hamiltonian arising from one-vector-gluon and long-range-scalar exchange could account for the mass splittings. To confirm the existence of $D_{1}^{\chi}$ it goes without saying that further analyses, including other experimental data with high statistics, are necessary in a more precise way.

Furthermore, in establishing the covariant level-classification scheme of meson systems it is important to examine the existence of the chiral scalar meson $D_{0}^{\chi}$ as well as $B_{0}^{\chi}$ and $B_{1}^{\chi}$ in the $B$ meson system. An analysis of the $B \pi$ mass spectrum, to study the existence of $B_{0}^{\chi}$, is in progress and its preliminary result has been presented. 3 )

\section{References}

[1] Ishida, S., Ishida, M., and Maeda, T., Prog. Theor. Phys. 104, 785-807 (2000).

[2] CLEO Collaboration, Phys. Letters B 331, 236-244 (1994); 342, 453(E) (1995).

[3] Ishida, M., and Ishida, S., these proceedings. 